\title{
Captive Breeding of Fire Shrimp (Lysmata debelius) under Sri Lankan Conditions
}

\author{
H.A.S.U. Hettiarachchi* and U. Edirisinghe ${ }^{1}$ \\ Postgraduate Institute of Agriculture \\ University of Peradeniya \\ Sri Lanka
}

\begin{abstract}
Lysmata debelius, commonly known as the Fire Shrimp or Scarlet Cleaner Shrimp is a highly demanded, pricy marine crustacean exported from Sri Lanka. However, information on captive breeding technology has not been reported so far in the country. Currently, majority of $\underline{L}$. debelius exported from Sri Lanka, is captured from the Indian Ocean, thus creating a threat of extinction of this indigenous species if not sustainably managed. Even in the global scale, the major obstacle in commercial production of $\underline{L}$. debelius is the poor survival of larvae accompanied by the lengthy larval life. Hence, this study was conducted with the objective of developing a captive breeding protocol and a larviculture technology as viable solutions to those problems. Purified natural sea water was used and natural environment was simulated. An effective broodstock diet was identified. Spawning chamber where water was conditioned with the diatom Chaetoceros gracillis was used for spawning. Larviculture was performed in glass aquariums of $108 \mathrm{~L}$. Feeding regime was developed for day 1-4, day 5-12, and day 13 to juvenile stage. Under the newly developed protocol, the duration of metamorphosis of larvae to juveniles was 50-90 days post hatching with the survival rate of $12.7 \pm 0.1 \%$ to $18.35 \pm 0.15 \%$, reporting the lowest larval duration and the highest survival rate reported so far for captive breeding of $\underline{L}$. debelius anywhere in the world. Survival of juvenile shrimps was 100\%. Experience of larviculture trials over the years was instrumental in developing this successful methodology. These results lay a firm foundation for future research and development of marine ornamental shrimp culture technology in Sri Lanka.
\end{abstract}

Keywords: Captive breeding, feeding regime, larval durations, larviculture, Lysmata debelius, survival rate

\section{INTRODUCTION}

Since the trading of marine ornamental species has become a highly profitable and a fastest growing industry in the world, the pressure on wild populations is building up creating a wide array of environmental implications. The marine segment of the aquarium industry still predominantly relies collection of wild specimens amounting to over $90 \%$ of the traded

\footnotetext{
${ }^{1}$ Department of Animal Science, Faculty of Agriculture, University of Peradeniya, Peradeniya, Sri Lanka

*Corresponding Author: udenihettiarachchi@gmail.com
} 
species being collected from coral reefs (Tlusty, 2002). Collection of wild marine ornamental species has been estimated to be responsible for $2 \%$ of coral reef destruction in the world (McManus et al., 1997).

During the last two decades, the market demand of these species has increased establishing a multi-million dollar industry worldwide (Wood, 2001; Hardin and Legore, 2005). As estimated, live marine animals worth of US\$ million 200-330 are traded annually throughout the tropics (Wabnitz, 2003). Developing mariculture technologies as well as improved biological studies will facilitate further growth in the trade of marine ornamental organisms.

L. debelius, being pricey and highly popular species indigenous to the Indo-Pacific region, plays a great role in the marine aquarium trade. The average individual selling price of $L$. debelius is $25 \mathrm{US} \$$, and thus make it a worthy candidate for marine ornamental trade and research (Palmtag and Holt, 2007). However, the prevailing situation of wild populations has urged the need to develop proper culture technologies (Calado et al., 2003). Therefore, cultivation efforts of $L$. debelius in warm marine waters have fetched a great concern across the globe.

Sri Lanka has no exception to global scenario with regard to exploitation of wild resource of L. debelius. Unscientific and selective removal of ornamental species, especially L. debelius from coastal regions of the country has affected their ecosystems which extend from the Trincomalee area in the east up to Puttlam in the north western coastal waters via Jaffna Peninsula of Sri Lanka (Edirisinghe, 1999). The exploitation pressure is becoming pronounced, at an exponential rate parallel to the growing demand. This situation has contributed to the endangered status of this fascinating species (Edirisinghe, 1999). The difficulty in captive breeding of this species is well known, and published information is scarce, especially in Sri Lankan conditions. Even though, there are limited information from other countries (Fletcher et al., 1995; Palmtag and Holt, 2001; Palmtag and Holt, 2007) about captive rearing, the applicability of those methods under Sri Lankan conditions is not well recognized.

As reported by Lin et al., (2002), the main constraint impairing the commercial culture of this valuable species is its poor survival rate during the lengthy larval period. In this context, development of an appropriate captive breeding methodology under Sri Lankan conditions would help to produce $L$. debelius in captivity and cater to the demand in the global aquarium trade while releasing the pressure on the wild stocks as well as the pressure on their immediate environment of coral reefs. Thus, the objective of this study was to develop a captive breeding protocol and larviculture technology of $L$. debelius with a simple technology under Sri Lankan conditions.

\section{MATERIALS AND METHODS}

\section{Research Location}

This research was conducted in a marine aquarium in Negombo, Western Province of Sri Lanka.

\section{Water Purification}

Purified sea water from the Indian Ocean was used in the culture system. In the purification process, sea water was pumped to a collecting sump and allowed 24-48 hours of sedimentation according to the level of turbidity. Then, the water was filtered through a high pressure sand filter and allowed for sedimentation in a second sump for 24 hours before 
filtering again through another high pressure sand filter. Finally, the filtered water was allowed to run through an Ultra -Violet sterilizer before using in the culture system.

\section{Water Quality Parameters}

Purified sea water in its natural condition was used in the culture system without regulating temperature, light, salinity or any other parameter by artificial means. The following water quality parameters were routinely measured and recorded. Water temperature was measured using a hand-held thermometer used in aquaculture. A hand-held salinity refractometer was used to record the salinity and specific gravity of water respectively. Liquid type water quality test kits designed for salt water aquariums were used to measure the nitrate and ammonia concentrations. Water $\mathrm{pH}$ was measured using a portable $\mathrm{pH}$ meter.

\section{Maturation System}

Adult $L$. debelius individuals from the wild stocks in Sri Lanka were collected from local divers of Puttlam. Selection of gravid females was avoided since they were susceptible to stress caused mortality. Ten pairs of $L$. debelius were used for the research. Since sexual dimorphism was not observed in L. debelius, and were simultaneous hermaphrodites, any two individuals could be selected as a pair. Initial body weight and total length of broodstock was $2.2 \pm 0.4 \mathrm{~g}$ and $5.25 \pm 0.35 \mathrm{~cm}$, respectively. Followed by acclimatization, shrimps were individually quarantined for 3 days before introducing to the maturation system. A pair of shrimps was introduced to each maturation tank.

The maturation system was consisted of a series of individual glass tanks in an indoor culture system where a pair of brooders was kept in each tank. The dimensions of each tank were 60 $\times 60 \times 60 \mathrm{~cm}$. Each broodstock tank had a $10 \mathrm{~mm}$ diameter inlet positioned by side on the top of the tank and an outlet of $25 \mathrm{~mm}$ at the opposite side. Outlet was located $4 \mathrm{~cm}$ below the top level. Therefore, full water volume of a broodstock tank was maintained at $200 \mathrm{~L}$. Outlet was covered with nylon mesh of $5 \mathrm{~mm}$ holes. Each tank was equipped with a valve to regulate water inflow.

Artificial lighting was not used in the system. Therefore, the system received natural sunlight as well as the natural light/dark regime. However, for optimum performance of the pair, excess illumination was avoided and natural reef habitat was simulated by placing blue polythene outside the glass walls of the broodstock tanks. In addition, two rock pieces were placed on the tank bottom to provide a cave like structure to facilitate their hiding and hanging behaviour (Figure 1). Each tank was provided with mild aeration.

Manual cleaning of tank bottom was practiced by siphoning once in the mornings every day. Each tank received approximately $25 \%$ total water exchange daily. Presence of moults and gravid shrimps were observed and recorded daily.

\section{Broodstock Feeding}

A mixed diet of cuttlefish and mussel meat in equal proportions was fed twice daily in the morning and evening at a rate of 5\% body weight. The amount fed was regulated according to acceptance of the feed by the pair. Frozen copepods were fed once a week by skipping a meal of the above feeding regime in order to boost the nutritional status of the broodstock. Aeration was stopped just before feeding and half an hour after feeding to facilitate the feed to sink to tank bottom. 


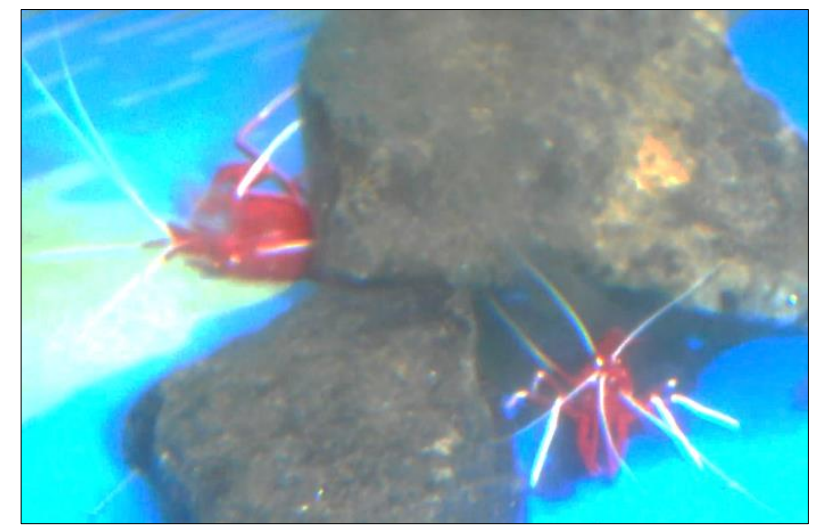

Figure 1. Pair of $L$. debelius in the cave structure inside the tank

\section{Preparation for Spawning}

For spawning, parent shrimps were moved to spawning tanks, which is a separate series of tanks with the dimensions of $60 \times 45 \times 45 \mathrm{~cm}$ covered all sides with blue polythene. Each tank was consisted with an inlet of $5 \mathrm{~mm}$ vinyl hose connected to the central water line. An outlet of $2.5 \mathrm{~cm}$ was located in the opposite direction of each inlet. Outlets were blocked with a fine mesh of $500 \mu \mathrm{m}$ to prevent escaping of larvae. Mild aeration was supplied to each tank using air stones. Outlet was located $5 \mathrm{~cm}$ below the top level of the tank. Therefore, a spawning tank holds $108 \mathrm{~L}$ at its full level.

A spawning chamber was made (Figure 2) with a rigid, black plastic mesh of 5 x $5 \mathrm{~mm}$ holes rolled to have the shape of a cylinder of $15 \mathrm{~cm}$ in diameter and $45 \mathrm{~cm}$ in height, which was tall enough to stand over the water surface of the spawning tank. The bottom end of the cylinder was fastened with the same type of mesh. This structure was externally supported to stand towards one of the sides of the spawning tank. A piece of rock was placed inside the spawning chamber to provide weight to restrict its movement inside the tank and support shrimps in the moulting process. Spawning chamber confined the parents within it and facilitated only the larvae to move out. It also facilitated the removal of parents the next morning.

Before introducing the parent shrimps to the spawning chamber, the diatom (Chaetoceros gracillis) was introduced to the spawning tank water at a density of 50,000 cells $/ \mathrm{mL}$ to condition the spawning tank water and to prevent starvation of larvae by the next morning.

In 10-12 days after observing eggs on swimmerets, both parents in the maturation tank were moved into the spawning chamber placed in the spawning tank. Instead of netting the parents, a wide mouth jar was placed on the tank bottom and only one shrimp was removed at a time. Extreme care was taken to avoid damage to eggs, when relocating brooding shrimp. This practice was performed during dusk. Irrespective to whether spawning occurred or not, the pair was returned to the maturation tank and normal feeding and changing of water were carried out early in the next morning. 


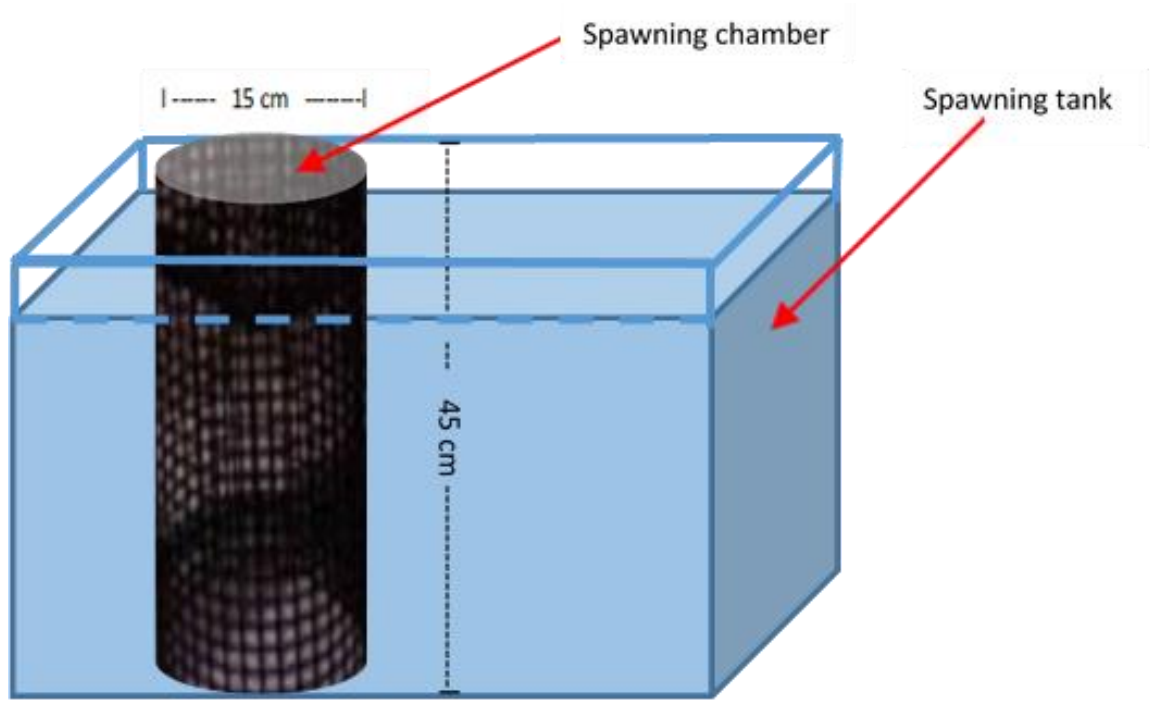

Figure 2. Diagrammatic representation of spawning set up

\section{Larval Rearing System}

The same spawning tank of $108 \mathrm{~L}$ was used as the larval rearing tank. Sides of the tank were covered with blue polythene except the front wall. Larviculture was carried out under natural sun light and ambient temperature without any artificial regulation. Soon after the removal of parent shrimps from the spawning tank, aeration was stopped for about half an hour and allowed the active larvae to migrate to surface. Then, larvae were siphoned off to a $2 \mathrm{~L}$ plastic cup using a $5 \mathrm{~mm}$ flexible tube. Larvae (zoea) were counted in 3 random samples and average was taken as the number of zoea per spawn. The bottom debris of the spawning tank was siphoned off using a flexible hose of $1.25 \mathrm{~cm}$ diameter. By this way, water level of the spawning tank was reduced to a height of $15 \mathrm{~cm}(36 \mathrm{~L})$ before the larvae were carefully returned to the same tank.

\section{Larviculture (1-4 Days)}

Initial water level of a larval rearing tank was $36 \mathrm{~L}$. Substrates were never used in larval rearing tanks where even the air stone was kept suspended off the bottom. Larval feeding commenced on the first day itself, after counting and replacing them in the larval rearing tank. First feeding was done with a mixed diet of diatom (Chaetoceros gracillis) and microalgae (Chlorella vulgaris) fed rotifers. Rotifers were enriched with green microalgae (C. vulgaris) twenty minutes before feeding. The density of feeding diatom was 10,000 cells/ml and fed once daily in the mornings. Rotifer (Bracheonus plicatillus ' $\mathrm{L}$ ' strain-125 $\mu \mathrm{m})$ was fed twice daily in the morning and evening. Feeding density was 5 rotifers $/ \mathrm{mL}$, and harvested rotifers were washed well before feeding. This feeding regime was continued for 3 days. On the $4^{\text {th }}$ day, density of rotifers was reduced to half and newly hatched brine shrimp (Artemia salina) fed with $C$. vulgaris was introduced at a density of 2 nauplii/mL. 
Water level of the larval rearing tank was increased by $5 \mathrm{~cm}$ per day starting from the third day of hatching and continued for 3 days. Microalgae cultures and rotifer cultures were maintained separately at the experimental site and strict hygiene was maintained.

\section{Larval Feeding and Water Exchange (Day 5-12)}

Newly hatched brine shrimp nauplii enriched with $C$. vulgaris 18 hours after hatching were used for feeding shrimp larvae between 4-12 days of age. Feeding density was 2 nauplii $/ \mathrm{mL}$. From the $10^{\text {th }}$ day onwards, cultured calanoid copepods at a density of $0.2 \mathrm{prey} / \mathrm{mL}$ were also added to the diet.

Water exchange in the larval rearing tanks was started from the $5^{\text {th }}$ day and continued routinely. During water exchange, one tenth of the water in the larval tank was reduced using a special siphoning device (Figure 3), and the floating debris adhered on the side walls were then removed with a sponge by dragging along the glass walls above the receded water line. Then the tank was refilled gradually with new water. Filling was carefully monitored

The special siphoning device was made with a PVC pipe of $10 \mathrm{~cm}$ in length and $50 \mathrm{~mm}$ in diameter. Holes were made by drilling and one end was closed with an end cap. A tight bag of $55 \mu \mathrm{m}$ nylon mesh was sewed to fit to the size of the PVC tube. The opened end was connected to a siphoning hose of $12 \mathrm{~mm}$ by using a reducing socket.

Aeration was stopped 15 minutes in advance when water was removed from the larval tank. Then, the siphoning device was submerged and a quick suction was applied at the open end of the flexible hose. While water level of the tank was receding, the device was gently shaken to avoid accidental trapping of larvae to the mesh. Before removing the device from the tank, the open end of the hose was closed with a finger and raised to stop suction.

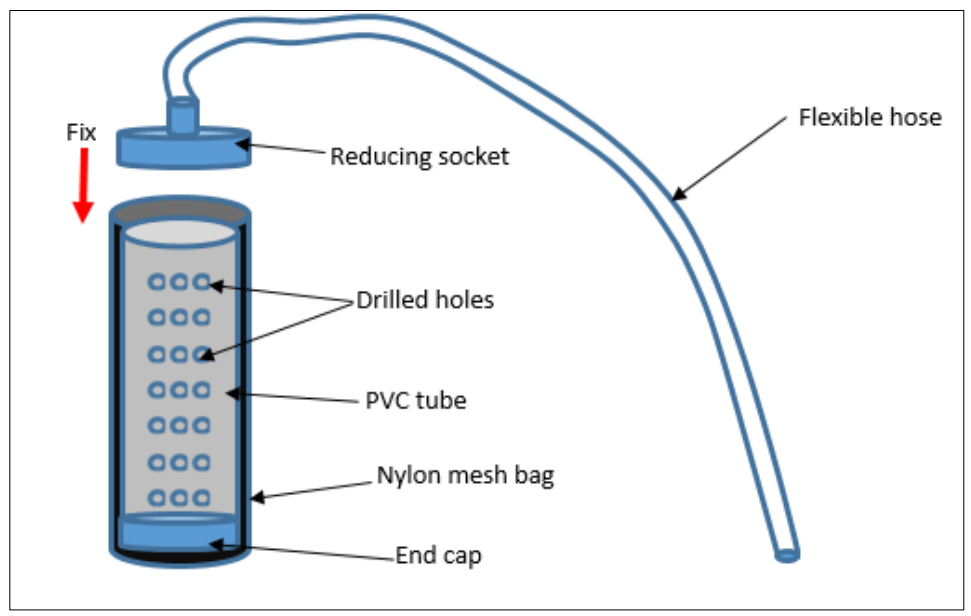

Figure 3. Diagrammatic representation of the siphoning device 


\section{Larval Feeding and Water Exchange (from Day 13 up to Metamorphosis to Juveniles)}

Amount of brine shrimp nauplii fed was reduced to 1 nauplii/mL and the pureed cuttlefish was introduced to enhance the nutritional status. Cultured live copepods at a density of 0.2 prey $/ \mathrm{mL}$ was also added to the diet. Bottom siphoning was practiced using a $10 \mathrm{~mm}$ tube to remove left over feed and debris. Water exchange was increased up to $20 \%$ during this period, and practiced routinely.

\section{Preparation of Cuttlefish for Feeding}

Fresh cuttlefish were well cleaned and cut into strips of about $15 \times 5 \mathrm{~cm}$ before freezing. Prior to feeding, a strip of frozen cuttlefish was grated with a household carrot grater while it was still in solid ice form. Then the grated pieces were washed well on a mesh and a small quantity of soften pieces just to suffice one piece per larva was used for feeding.

\section{Care of Juveniles}

Juvenile shrimps were those who completed the spidery shape larval life and metamorphosed to the appearance of miniature adults. By that time, they were 50-90 days of age. As soon as metamorphosed, the juvenile shrimps were individually collected using a concave spoon and were carefully transferred to nursery tanks. This routine was carried out daily in the morning. Nursery tanks were rectangular glass tanks of $120 \times 55 \times 45 \mathrm{~cm}$, carrying $10 \mathrm{~mm}$ diameter inlet positioned by side on the top of the tank and an outlet of $25 \mathrm{~mm}$ at the opposite side. Outlet covered with nylon mesh of $3 \mathrm{~mm}$ holes, was located $4 \mathrm{~cm}$ below the top level. Therefore, full water level of a nursery tank was 275 L. Natural sunlight and mild aeration were provided in each nursery tank. Cave like structures made from PVC pipes were the only substrate used in the tank. A PVC pipe of $10 \mathrm{~cm}$ in diameter was cut in to pieces of $6 \mathrm{~cm}$ in length and each piece was split in to two in length-wise for this purpose.

Feeding with brine shrimp nauplii was gradually stopped in nursery tanks. However, feeding grated cuttlefish and copepods was continued as usual for another 3 days. In addition, commercial shrimp feed was introduced and the amount fed was gradually increased up to ad libitum feeding. Granular size of commercial shrimp feed was $1 \mathrm{~mm}$ and the nutritional composition was, crude protein $60 \%$, crude fat $10 \%$, crude fiber $1 \%$, and moisture $8 \%$.

Water exchange was practiced routinely as described previously by allowing the tank to fill with new water, while flowing through the outlet to give nearly $25 \%$ daily water exchange.

\section{Data Collection}

Four deaths were recorded during the breeding trial in four maturation tanks. Deaths were due to lost antenna or damaged appendages, which could be a result of aggressive behavior during pair formation. The remaining shrimps were mixed in order to form two new pairs. As indicated by Calado et al. (2007), individuals with agonistic behavior could be allowed to pair by physically isolating them using a movable plastic mesh inside each tank, and isolating the pair for a while still having access to feel the presence of each other and to develop the bond between them.

Even under optimum conditions provided, there were certain limitations observed during breeding trial viz. only a negligible number of larvae were produced by four spawners, and 
spawning was not consecutive. They also had discarded moults, which were still carrying embryos. Data of those four shrimps were not considered.

Therefore, spawning data of 12 successful spawners up to 5 batches were collected during the experimental period. Larval duration was calculated by the number of days taken to complete the larval life. That was the duration from hatching up to metamorphosis to juvenile shrimps. Survival rate was estimated by counting the number of larvae metamorphosed to juveniles.

\section{RESULTS AND DISCUSSION}

During the experimental period, water temperature varied from $28-30{ }^{\circ} \mathrm{C}$ and the salinity from 30-37 ppt. Water $\mathrm{pH}$ and specific gravity varied between 7.7-8.5 and 1.025-1.027, respectively. Nitrate concentration remained below $40 \mathrm{mg} / \mathrm{L}$ and the ammonia concentration was less than $0.8 \mathrm{mg} / \mathrm{L}$.

The observed spawning interval of this study was 11-13 days, and spawning occurred in the night, between 1.00-3.00 a.m. The average number of larvae per spawner was $845 \pm 255$. It was a lower value compared to the data published by Calado et al. (2007), which was $1633 \pm$ 282 under different environmental conditions. However, since the size of adult shrimps was not indicated, it was unable to get a clear understanding of whether it reflects size differences.

In the present study, the initial body sizes of parent shrimps had slight differences and the average number of larvae released per spawner directly correlated to the weight of parent shrimp (Table 1). However, weight differences in the shrimps having similar body length may be due to the differences in individual ovarian conditions.

Table 1. Body Size of spawners and spawning details

\begin{tabular}{cccc}
\hline Spawner No: & Total Length $(\mathbf{c m})$ & Weight $(\mathbf{g})$ & $\begin{array}{c}\text { Number of larvae released } \\
\text { in 5 batches (Mean } \pm \text { SD) }\end{array}$ \\
\hline 1 & 4.9 & 1.8 & $670 \pm 80$ \\
2 & 5.1 & 1.8 & $694 \pm 46$ \\
3 & 5.3 & 2.0 & $707 \pm 73$ \\
4 & 5.2 & 1.8 & $722 \pm 78$ \\
5 & 5.3 & 1.9 & $725 \pm 75$ \\
6 & 5.4 & 2.4 & $730 \pm 30$ \\
7 & 5.0 & 1.8 & $765 \pm 65$ \\
8 & 5.5 & 2.2 & $800 \pm 80$ \\
9 & 5.6 & 2.4 & $889 \pm 51$ \\
10 & 5.4 & 2.6 & $908 \pm 82$ \\
11 & 5.5 & 2.5 & $920 \pm 80$ \\
12 & 5.6 & 2.6 & $1000 \pm 100$ \\
\hline
\end{tabular}




\section{Larval Life and Durations}

The appearance of newly hatched L. debelius larvae (zoea) was quite different from the adult shrimp. Energetic zoea were highly phototrophic and about 1-2 mm in size. Most of the body parts of zoea were rudimentary. They did not possess eye stalks. They exhibited many morphological changes throughout the larval life. In 48 hours, post-hatching, moulting of zoea 1 to zoea 2 stage was evident by numerous tiny exoskeletons floating on the water surface. A pair of uropods at the sides of the tail was visible in 5-6 days. At this age, larvae were 2-3 $\mathrm{mm}$ in size.

By the age of 7-8 days, they reached the size of 3-4 mm and a pair of spear legs was visible. Until metamorphosis, these spear legs increased in the length to reach twice the body length giving a spidery appearance to the larva. By that age, the average body length of larvae was $1 \mathrm{~cm}$ in size. During latter stage of the larval life, these spidery shape larvae oriented in head down position (Figure 4).

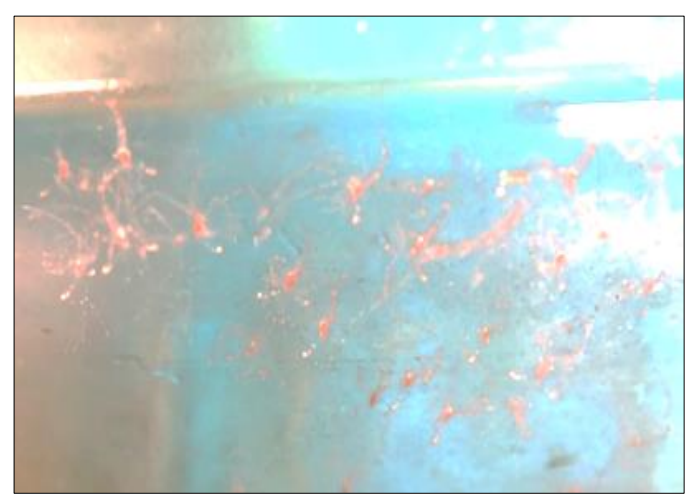

Figure 4. Larvae orient in head down position

Larvae turned to miniature adults in 50-90 days post-hatching. This metamorphosis occurred only during night. Juveniles were pale in colour for the first 3 days, but resembled miniature adults (Figure 5). They were on the side walls clinging off the bottom.

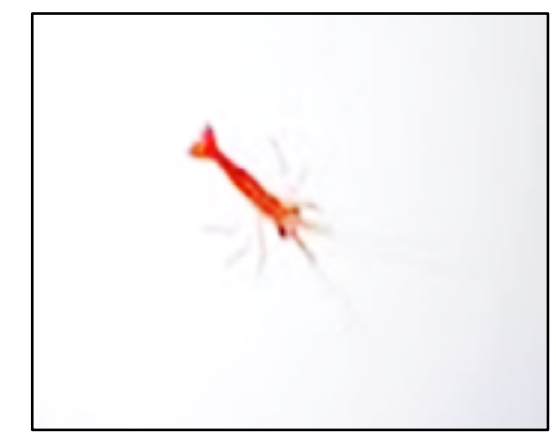

Figure 5. Juvenile immediately after metamorphosis 
Pale colour juveniles turned to brilliant red colour gradually resembling their parents, and they exhibited schooling and hiding behaviour inside the PVC cave like structures provided as the substrate (Figure 6).
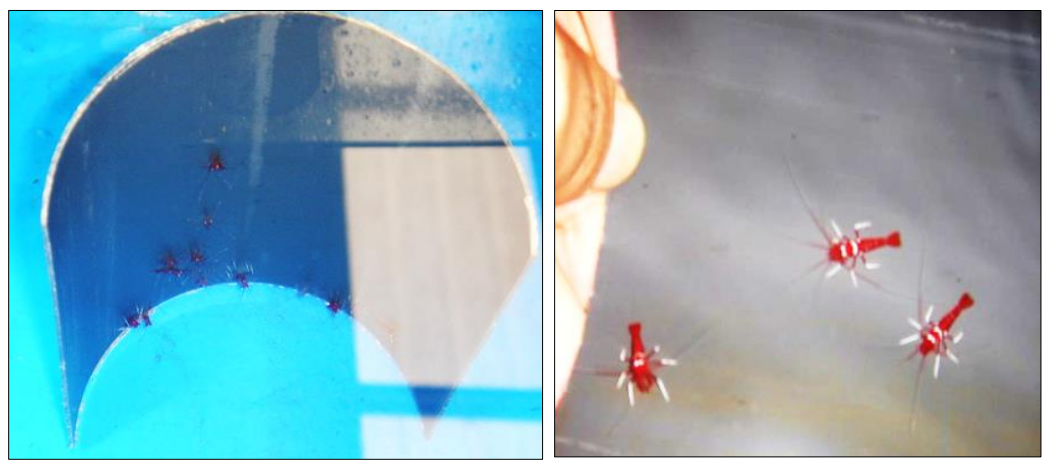

Figure 6. Juveniles hiding inside PVC cave structure

The larval duration observed under Sri Lankan conditions (50-90 days) was much shorter than those reported in the literature (75-158 days). The reasons for the shorter larval duration in the present study may be due to the nutrition provided in larval diets, management practices, and the specific climatic conditions in Sri Lanka, which favored the growth of $L$. debelius larvae. However, a whole batch of larvae did not metamorphose to juveniles in one night. It started from a few and continued up to a period of 1.5 months. According to that, the shortest larval life was 50 days and the maximum was 90 days. By that time, those metamorphosed to juveniles at first, had reached 2-3 cm. Fletcher et al. (1995), indicated that metamorphosis of $L$. debelius occurred in 77-105 days in their experiments while Palmtag and Holt (2001) reported that metamorphosis of larvae occurred in 75-158 days. These differences in larval durations under different conditions compared to Sri Lankan condition are illustrated in Figure 7.

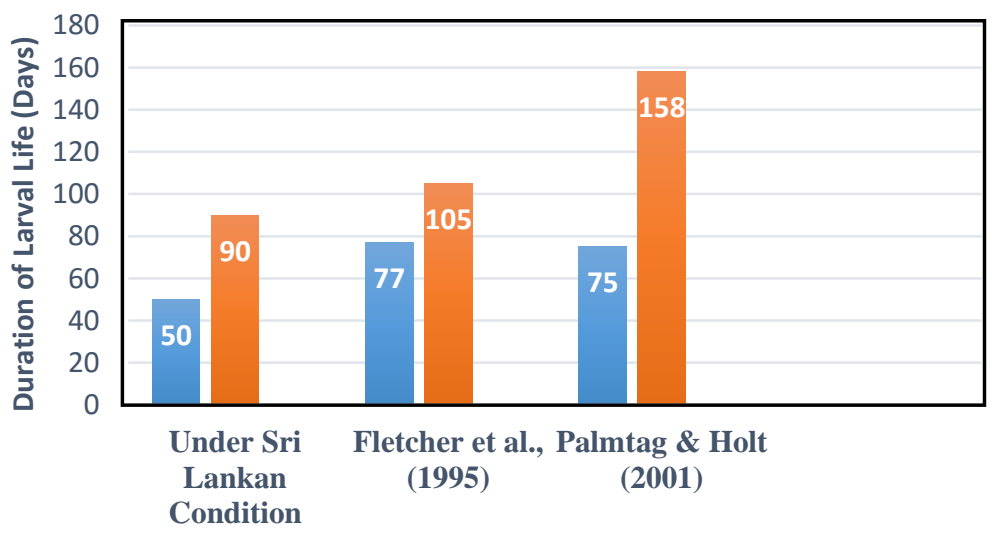

Figure 7. Duration of larval life under different conditions 


\section{Survival Rate}

Survival rate is the average number of zoea metamorphosed to juveniles out of a batch of zoea. In this research, $12.7 \pm 0.1 \%$ to $18.35 \pm 0.15 \%$ survival was obtained from 12 spawners in 5 spawning each (Table 2). Juvenile shrimps were quite hardy and the mortalities were not observed. However, careful handling was essential to avoid possible damages when they were transferred to nursery tanks as well as during siphoning off the tank bottoms.

Palmtag and Holt (2001) have estimated that approximately $10 \%$ of the spawn had metamorphosed to juveniles in their trial. According to Palmtag and Holt (2007), the highest rate of survival to the juvenile stage resulted from the feeding protocol that included shrimp muscle in pureed form was $9.8 \pm 1.2 \%$.

\section{Table 2. Larval survival rates}

\begin{tabular}{ccc}
\hline Spawner No: & $\begin{array}{c}\text { Number of larvae released } \\
(\text { Mean } \pm \text { SD) }\end{array}$ & $\begin{array}{c}\text { Larval survival rate } \\
(\text { Mean } \pm \text { SD) }\end{array}$ \\
\hline 1 & $670 \pm 80$ & $17.40 \pm 0.60 \%$ \\
2 & $694 \pm 46$ & $15.85 \pm 0.35 \%$ \\
3 & $707 \pm 73$ & $12.70 \pm 0.10 \%$ \\
4 & $722 \pm 78$ & $15.50 \pm 0.20 \%$ \\
5 & $725 \pm 75$ & $18.30 \pm 0.10 \%$ \\
6 & $730 \pm 30$ & $18.25 \pm 0.35 \%$ \\
7 & $765 \pm 65$ & $16.20 \pm 0.30 \%$ \\
8 & $800 \pm 80$ & $18.35 \pm 0.15 \%$ \\
9 & $889 \pm 51$ & $18.30 \pm 0.30 \%$ \\
10 & $908 \pm 82$ & $18.15 \pm 0.35 \%$ \\
11 & $920 \pm 80$ & $17.20 \pm 0.40 \%$ \\
12 & $1000 \pm 100$ & $18.25 \pm 0.35 \%$ \\
\hline
\end{tabular}

\section{CONCLUSIONS}

The present study has proven that captive breeding of $L$. debelius is possible under Sri Lankan conditions with 50 - 90 days larval duration and at $12.7 \pm 0.1 \%$ to $18.35 \pm 0.15 \%$ survival rates.

\section{REFERENCES}

Calado, R. J., Lin, A. L., Rhyne, R., Araujo and Narciso, L. (2003). Marine ornamental decapods - popular, pricey, and poorly studied. J. Crustacean Biol. 23(4), 963 - 973.

Calado, R., Vitorino, A., Dionisio, G., and Dinis, M. T. (2007). A recirculated maturation system for marine ornamental decapods. Aquaculture. 263, 68 - 74. 
Edirisinghe, U. (1999). Ornamental fisheries as a livelihood for coastal Regions. pp. 70-73. In: Gunasena, H.P.M., Kotagama, H.B., and Wickramasuriya, H.V.A. (Ed.) Coastal Resources Management in Sri Lanka. International Development Research Centre, New Delhi, India.

Fletcher, D. J., Kotter, I., Wunsch, M., and Yasir, I. (1995). Preliminary observations on the reproductive biology of ornamental cleaner prawns. International Zoology Yearbook. 34, 73 77.

Hardin, M. and Legore, R. (2005). Development of management policy for the marine ornamental fish and invertebrate fishery in Puerto Rico: A case study. Rev. Biol. Trop. 53, $139-144$.

Lin, J., Zhang, D., and Rhyne, A. L. (2002). Broodstock and Larval Nutrition of Marine Ornamental Shrimp. Florida Institute of Technology, $150 \mathrm{~W}$, University Blvd., Melbourne, FL 32901, USA.

McManus, J. W., Reyes, R. B., and Nanola, C. L. (1997). Effects of some destructive fishing methods on coral cover and potential rate of recovery. J. Environ. Manage. 21, 69 - 78.

Palmtag, M. R. and Holt, G. J. (2001). Captive rearing of fire shrimp (Lysmata debelius). A Texas Sea Grant College Program Research Report. National Oceanic and Atmospheric Administration, U.S. Department of Commerce, Washington, DC, USA.

Palmtag, M. R., and Holt, G. J. (2007). Experimental Studies to Evaluate Larval Survival of the Fire Shrimp, Lysmata debelius. J. World Aquac. Soc. 38 (1), $102-113$

Tlusty, M. (2002). The benefits and risks of aquacultural production for the aquarium trade. Aquaculture. 205, 203 - 219.

Wabnitz, C., Taylor, M., Green, E. and Razak, T. (2003). From ocean to aquarium. UNEPWCMC, Cambridge, UK.

Wood, E. M. (2001). Collection of coral reef fish for aquaria: global trade, conservation issues and management strategies. Marine Conservation Society UK. 